\title{
A Robust Two-Stage Stochastic Location-Routing-Inventory Model for Perishable Items
}

\author{
Shima HARATI*, Emad ROGHANIAN, Ashkan HAFEZALKOTOB, Amir Abbas SHOJAIE
}

\begin{abstract}
The aim of this study is to develop a robust two-stage stochastic location-routing-inventory model for perishable items. The proposed model is implemented in a two-stage structure. The first-stage decisions determine the establishment of distribution centres and the second-stage decisions identify the other variables of the problem. In order to reduce the effect of different scenarios on the outputs of the problem, the two-stage model is developed to a robust model. Two variability criteria called 'Partial Lower Deviation from Mean' (PLDM) and 'Partial Lower Deviation from Target' (PLDT) are considered for the problem. This robust model can manage the variability of different scenarios considering the variability needed for the problem. The summary of the results of the models indicate that the supply cost, the setup cost, the vehicle supply cost, and the production cost comprise $55 \%, 28 \%, 3 \%$, and $14 \%$ of the total costs of the supply chain, respectively. Similarly, the ratio of net profit margin to the total revenue of the supply chain derived from the division of the objective function by the revenue function is $15 \%$. Among free, fresher first, older first, and mixed policies, the free policy provides the decision maker with more profit than the other three policies since it imposes less constraints on the model.
\end{abstract}

Keywords: Inventory; Locating; Perishable items; Robust two-stage stochastic model; Routing

\section{INTRODUCTION}

This study considers a three-level supply chain for dairy products. The first level is the raw milk supplier. The second level of the supply chain is the dairy plants which receive milk from the suppliers, process and send it to the third level of the supply chain which is the consumer centres. One of the main objectives of this study is to establish a proper connection and routing among supply chain levels along with locating the distribution facilities by considering transportation. In the problem under study, considering the dispersion of raw milk production and consumer centres, it is particularly important to locate the middle level, that is, the dairy supplier. In this study, the establishment of production centres is considered as a variable and is determined according to the other parameters of the problem. Since decisions about the establishment of a dairy production plant are related to strategic-tactical levels and routing and determining the transfer rate are related to tactical-operational levels, simultaneous decision making for all the variables is not possible. To solve this problem in the current research, a two-stage decision-making method is used. The decisions are first made in relation to the establishment and setup of the dairy plant and in the next stage other routing-inventory decisions are made. Due to the fact that the considered items are perishable, the model has been studied in the periodic mode. By passing from one period to the next, if a product remains in the warehouse, its shelf life increases by one unit. Different shelf lives of the items can have different revenues for the system.

On the other hand, the idea of perishable goods in supply chains has been studied by numerous researchers such as Liu et al. [1], Shah [2], Onggo et al. [3], Yang and Wee [4], and Rau et al. [5], Yavari and Geraeli [6]. Inventory management by the seller can be mentioned as one of the effective strategies in this field. In this strategy, by considering the need to an inventory renewal period for the consumers, the manufacturer decides about the time and quantity of inventory renewal as well as the system. Given the importance of the supply chain and perishable goods, some researchers have considered the integrity of the chain along with perishable goods. Seyedhosseini and
Ghoreyshi [7] presented an integrated model for the production and distribution of perishable items considering inventory and routing problems.

Yu et al. [8] addressed the perishability of goods in the integrated two-level supply chain and developed an integrated model for perishable goods. In their proposed model, perishability has been considered for the final product, retailer demand has been assumed to be deterministic and constant, and the optimum values have been determined using a heuristic and metaheuristic hybrid approach in order to maximize the supply chain profit. Shaabani and Kamalabadi [9] presented an efficient simulated annealing model for the supply chain with a few consumers who purchase several perishable goods. Hsu et al. [10] presented a routing problem with a time window for perishable items. Seyedhosseini et al. [11] studied the problem of perishable items with stochastic demand for a supply chain with two levels. The main objective of the current study is to present a robust two-stage stochastic location-routing-inventory model for perishable items. Chao et al. [12] presented a mixed-integer programming model for location-routing-inventory problem with timewindows problem and solved it by a hybrid heuristic algorithm. Khalili-Damghani [13] proposed a bi-objective location-routing mixed integer model to distribute perishable products in supply chains and solved it by NSGA-II and $\varepsilon$ - constraint.

The following research gaps were identified after reviewing previous studies in this paper:

- Although the time horizons of most of the decisionmaking models are infinite, these models have been considered in the framework of economic ordering or economic production models.

- A two-stage model that considers both strategic and operational decisions simultaneously has not been investigated in the literature so far.

- Most of the previous studies have a specific demand function, while the specific characteristics of perishable items make a simple demand function insufficient for them. Most of the demand functions in the literature depend on the time, price, or expiration period, whereas this research offers a flexible approach for the type of demand. 
- The problems that have considered the relationship between inventory and routing regarding perishable items are very limited and few. The purpose of the current study is to investigate this topic carefully.

\section{MATHEMATICAL MODEL}

\subsection{Assumptions}

In this paper, 13 assumptions are considered. These assumptions are:

1. The studied chain is multilevel and includes the levels of supply, plant, and demand.

2. The chain under study is multi-period and multi-product.

3. The locations of the suppliers and retailers are already known, but the location of the plant is determined by the model.

4. Routing is done among all levels.

5. The routing problem is multi-depot with a certain capacity.

6. The retailer/supplier index with the number 1 represents the plant.

7. The vehicles and centres have a certain capacity.

8 . The vehicles are considered as heterogeneous.

9. The vehicles used for collecting milk from the suppliers and the vehicles used for delivering the product to the customers are of different types.

10. The products delivered to the customers have shelf lives (age).

11. Storing the product in the plant's warehouse for the next period has been considered possible.

12. A hard time window has been considered for collecting milk from the suppliers.

13. At any time period, the price of the delivered product to customers varies according to its shelf life.

\subsection{Indicators}

The model indicators are as follows:

Product - $j$

Supplier - $a, a^{\prime}$

Plant - $m$

Retailer - $n, n^{\prime}$

Vehicle for purchasing milk - $p$

Vehicle for delivering the product $-q$

Product age $-g$

Time period $-t$

Scenario $-s$

\subsection{Parameters} follows:

The parameters of mathematical model are shown as $C P T Y_{\text {ats }}^{\text {sup }}$ - The capacity of supplier $a$ for supplying milk in time period $t$ under scenario $s$.

$C P T Y_{j m t}^{m a n}$ - The capacity of plant $m$ for product $j$ in time period $t$.

$C P T Y_{p}^{R-v e h}$ - The capacity of milk-collecting vehicles $p$. $C P T Y_{q}^{D-v e h}$ - The capacity of vehicles $q$ used for delivering the product to the customers. $\omega_{a^{\prime} a}^{\text {sup }}$ - The distance between supplier $a$ and supplier $a^{\prime}$.

$\omega_{m a}$ - The distance between plant $m$ and supplier $a$.

$\varpi_{n^{\prime} n}^{r e t}$ - The distance between retailer $n$ and retailer $n^{\prime}$.

$\varpi_{m n}$ - The distance between plant $m$ and retailer $n$.

$\omega T_{p a^{\prime} a}^{\text {sup }}$ - The time period between supplier $a$ and supplier $a^{\prime}$ by vehicle $p$.

$\omega T_{p m a}$ - The time period between plant $m$ and supplier $a$ by vehicle $p$.

$\xi$ - The time window for collecting milk from the suppliers.

$O P M N_{m}$ - The setup costs of plant $m$.

$O P R_{p}$ - The cost of purchasing vehicle $p$.

$O P D_{q}$ - The cost of purchasing vehicle $q$.

$P R C_{a t}$ - The price of purchasing each unit of milk from supplier $a$ in time period $t$.

$P R C C_{j g t}$ - The price of selling each unit of product $j$ with age $g$ to the customers in time period $t$.

$P H_{\text {jgmt }}$ - The cost of holding each unit of product $j$ with age $g$ in the warehouse of plant $m$ in time period $t$.

$P M_{j m t}$ - The cost of producing each unit of product $j$ by plant $m$ in time period $t$.

$P R_{j}$ - The amount of milk required for producing each unit of product $j$.

demand $_{j n t s}$ - The demand of retailer $n$ for product $j$ in time period $t$ under scenario $s$.

$\lambda_{p}^{R}$ - The amount of fuel consumed by vehicle $p$ per unit distance.

$\lambda_{q}^{D}$ - The amount of fuel consumed by vehicle $q$ per unit distance.

$P R O_{s}$ - The probability of an event under scenario $s$.

$F$ - The price of each fuel unit.

bigm - A very big number.

\subsection{Variables}

The variables of mathematical model are as follows: $X_{p s}^{R}\left\{\begin{array}{l}1 \\ 0\end{array}\right.$ - Binary, If vehicle $p$ is purchased under scenario $S$. $X_{q s}^{D}\left\{\begin{array}{l}1 \\ 0\end{array}\right.$ - Binary, If vehicle $q$ is purchased under scenario $S$. $Y_{\text {pa'ats }}^{R}\left\{\begin{array}{l}1 \\ 0\end{array}\right.$ - Binary, If vehicle $p$ visits supplier $a$ after visiting supplier $a^{\prime}$ under scenario $s$ in time period $t$.

$Y_{q n^{\prime} n t s}^{D} \begin{cases}1 & - \text { Binary, If vehicle } q \text { visits retailer } n \text { after } \\ 0 & \end{cases}$ visiting retailer $n^{\prime}$ under scenario s in time period $t$.

$Z_{p m s}^{R}\left\{\begin{array}{l}1 \\ 0\end{array} \quad\right.$ - Binary, If vehicle $p$ is allocated to plant $m$ under scenario $s$. 
$Z_{q m s}^{D}\left\{\begin{array}{l}1 \\ 0\end{array}\right.$ - Binary, If vehicle $q$ is allocated to plant $m$ under scenario $s$.

$X_{m}\left\{\begin{array}{l}1 \\ 0\end{array}\right.$ - Binary, If plant $m$ is set up.

$Y_{\text {pamts }}$ - Positive, The amount of purchased milk by vehicle $p$ belonging to plant $m$ from supplier $a$ in time period $t$ under scenario $s$.

$Z_{\text {jgqmnts }}$ - Positive, The amount of product $j$ with age $g$ delivered to customer $n$ by vehicle $q$ belonging to plant $m$ in time period $t$ under scenario $s$.

$\lambda_{\text {jmts }}$ - Positive, The amount of product $j$ produced by plant $m$ in time period $t$ under scenario $s$.

$\alpha_{\text {jgqnts }}$ - Positive, The amount of product $j$ with age $g$ in vehicle $q$ while leaving the location of customer $n$ in time period $t$ under scenario $s$.

$A_{\text {pats }}$ - Positive, The arrival time of vehicle $p$ to the location of supplier $a$ in time period $t$ under scenario $s$.

$S T_{\text {jgmts }}$ - Positive, The amount of product $j$ with age $g$ in the warehouse of plant $m$ in time period $t$ under scenario $s$.

\subsection{Objective Function}

The objective function minimizes the costs. These costs include plant setup costs, the costs of purchasing milk-collecting vehicles, the costs of purchasing vehicles for delivering the product to customers, the costs of purchasing milk from the suppliers, the costs of holding raw materials in the plant, production costs in the plant, and the costs of fuel consumed by milk-collecting and product delivery vehicles.

$$
\begin{aligned}
& \text { Max } Z^{\text {benefit }}=\sum_{j, g, q, m, n, t, s} P R O_{s} \times P R C C_{j g t} \times Z_{j g q m n t s}- \\
& -\left(\sum_{m} O P M N_{m} \times X_{m}+\sum_{s} P R O_{s} \times\left(\sum_{p} O P R_{p} \times X_{p s}^{R}+\right.\right. \\
& +\sum_{q} O P D_{q} \times X_{q s}^{D}+\sum_{p, a, m, t} P R C_{a t} \times Y_{p a m t s}+\sum_{j, g, m, t} P H_{j g m t} \times \\
& \times S T_{j g m t s}+\sum_{j, g, q, m, n, t} P M_{j m t} \times Z_{j g q m n t s}+F \times \sum_{p, a^{\prime}>1, a>1, t} \lambda_{p}^{R} \times \omega_{a^{\prime} a}^{s u p} \times \\
& \times Y_{p a^{\prime} a t s}^{R}+F \times \sum_{p, m, a,>1, t} \lambda_{p}^{R} \times \omega_{m a} \times Z_{p m s}^{R} \times\left(Y_{p 1 a t s}^{R}+Y_{p a 1 t s}^{R}\right)+ \\
& F \times \sum_{q, n^{\prime}>1, n>1, t} \lambda_{q}^{D} \times \omega_{n^{\prime} n}^{r e t} \times Y_{q n^{\prime} n t s}^{D}+F \times \sum_{q, m, n,>1, t} \lambda_{q}^{D} \times \varpi_{m n} \times \\
& \left.\left.\times Z_{q m s}^{D} \times\left(Y_{q 1 n t s}^{D}+Y_{q n 1 t s}^{D}\right)\right)\right)
\end{aligned}
$$

Subjected to:

$$
\begin{aligned}
& S T_{j g m t s}=S T_{j(g-1) m(t-1) s}-\sum_{q, n} Z_{j g q m n t s} \quad \forall j, g>1, m, t>1, s \\
& S T_{j g m t s}=\lambda_{j m t s}-\sum_{q, n} Z_{j g q m n t s} \quad \forall j, g=1, m, t, s
\end{aligned}
$$

$$
\begin{aligned}
& \frac{\sum_{p, a} Y_{\text {pamts }}}{P R_{j}}=\sum_{g, q, n} Z_{j g q m n t s} \quad \forall j, m, t, s \\
& \sum_{g, q, m} Z_{j g q m n t s} \geq \text { demand }_{j n t s} \quad \forall j, n, t, s \\
& \sum_{p, m} Y_{\text {pamts }} \leq C P T Y_{a t s}^{\text {sup }} \quad \forall a, t, s \\
& \sum_{p, a} Y_{\text {gpamts }} \leq C P T Y_{j m t}^{\text {man }} \quad \forall j, m, t, s
\end{aligned}
$$$$
\sum_{g, q, n} Z_{j g q m n t s} \leq C P T Y_{j m t}^{m a n} \quad \forall j, m, t, s
$$$$
\sum_{a} Y_{\text {pamts }} \leq C P T Y_{p}^{R-v e h} \quad \forall p, m, t, s
$$$$
\sum_{g, j, n} Z_{j g q m n t s} \leq C P T Y_{q}^{D-v e h} \quad \forall q, m, t, s
$$

$\sum_{a^{\prime}} Y_{p a^{\prime} a t s}^{R} \leq 1 \quad \forall p, a, t, s$

$\sum_{n^{\prime}} Y_{q n^{\prime} n t s}^{D} \leq 1 \quad \forall q, n, t, s$

$\sum_{a^{\prime}} Y_{p a^{\prime} a t s}^{R}=\sum_{a^{\prime}} Y_{p a a^{\prime} t s}^{R} \quad \forall p, a, t, s$

$\sum_{n^{\prime}} Y_{q n^{\prime} n t s}^{D}=\sum_{n^{\prime}} Y_{q n n^{\prime} t s}^{D} \quad \forall q, n, t, s$

$$
\begin{aligned}
& A_{p a t s}+\left(1-Y_{p a^{\prime} a t s}^{R}\right) \times b i g m \geq A_{p a^{\prime} t s}+\omega T_{p a^{\prime} a}^{s u p} \\
& \forall p, a^{\prime}, a>1, t, s
\end{aligned}
$$

$\xi+\left(1-Y_{p a 1 t s}^{R}\right) \times b i g m \geq A_{p a t s}+\omega T_{p m a} \times Z_{p m s}^{R}$

$\forall p, m, a>1, t, s$

$\alpha_{j q n^{\prime} t s}+\left(1-Y_{q n^{\prime} n t s}^{D}\right) \times b i g m \geq \alpha_{j q n t s}+Z_{j q m n t s}$

$\forall j, q, m, n^{\prime}, n>1, t, s$

$\alpha_{j q 1 t s} \geq \sum_{n} Z_{j q m n t s} \quad \forall j, q, m, t, s$

$Y_{\text {pamts }} \leq$ bigm $\times \sum_{a^{\prime}} Y_{p a^{\prime} a t s}^{R} \quad \forall p, a, m, t, s$

$Z_{\text {jgqmnts }} \leq \operatorname{bigm} \times \sum_{n^{\prime}} Y_{q n^{\prime} n t s}^{D} \quad \forall j, g, q, m, n, t, s$

$Y_{\text {pamts }} \leq$ bigm $\times Z_{p m s}^{R} \quad \forall p, a, m, t, s$ 


$$
\begin{aligned}
& Z_{\text {jgqmnts }} \leq b i g m \times Z_{q m s}^{D} \quad \forall j, g, q, m, n, t, s \\
& Y_{\text {pamts }} \leq b i g m \times X_{m} \quad \forall p, a, m, t, s \\
& Z_{j g q m n t s} \leq b i g m \times X_{m} \quad \forall j, g, q, m, n, t, s \\
& Z_{p m s}^{R} \leq X_{p s}^{R} \quad \forall p, m, s \\
& Z_{q m s}^{D} \leq X_{q s}^{D} \quad \forall q, m, s \\
& \sum_{p} Z_{p m s}^{R} \leq b i g m \times X_{m} \quad \forall m, s \\
& \sum_{q} Z_{q m s}^{D} \leq b i g m \times X_{m} \quad \forall m, s \\
& \sum_{m} Z_{p m s}^{R} \leq 1 \quad \forall p, s \\
& \sum_{m} Z_{q m s}^{D} \leq 1 \quad \forall q, s \\
&
\end{aligned}
$$

The balance of the product inventory in the plant's warehouse has been considered by Eq. (1) and Eq. (2). Eq. (3) calculates the amount of milk consumed in each period for manufacturing the products. Eq. (4) guarantees the satisfaction of demand. Eq. (5) to Eq. (9) ensure that the capacities of the supplier, plant, milk-carrying vehicles, and product-carrying vehicles are not exceeded, respectively. Each vehicle will visit each supplier and each retailer once in each time period. Eq. (10) and Eq. (11) are for the suppliers and retailers, respectively. Based on Eq. (12), if a vehicle enters a supplier centre, it must leave the centre. Moreover, if a vehicle visits a retailer, it must leave the retailer as included in Eq. (13). Eq. (14) and Eq. (15) respectively ensure that the subtour is eliminated and the sequence of supplier visit by the vehicles is determined. Moreover, Eq. (16) has been used to ensure that the time window for the return of milk-carrying vehicles to the plant is not exceeded. Moreover, Eq. (16) is the subtour elimination constraint and Eq. (17) determines the sequence of product-carrying vehicles to deliver the products to the retailers.

One of the conditions for collecting milk from the suppliers and delivering the products to the retailers is that they be visited by vehicles. These conditions for the suppliers and retailers are included in Eq. (18) and Eq. (19), respectively. Another condition for collecting milk from the suppliers and delivering the products to the retailers is that the vehicles be allocated to the plants. This condition for milk-collecting and product delivery vehicles has been included in Eq. (20) and Eq. (21), respectively. If a plant is not operational, milk should not be delivered to it or products be sent from it to the customers. This condition has been considered in Eq. (22) and Eq. (23), respectively. If a vehicle is not purchased, it must not be allocated to the plant. This condition for milk-collecting vehicles and for delivery vehicles is included in Eq. (24) and Eq. (25), respectively. Another condition is that if a plant is not operational, no vehicles should be allocated to it. This condition for receiving milk and product delivery has been considered in Eq. (26) and Eq. (27), respectively. Each vehicle must be allocated to one distribution centre at most. For milk-collecting vehicles Eq. (28) and for product delivery vehicles Eq. (29) have been considered.

\subsection{Solution Approach \\ 2.6.1 Developing the Two-Stage Stochastic Model Into a Robustness Model}

In practice, stochastic programming and robust optimization are used to manage the uncertainties and limitations in the relevant literature. The robust counterpart of the general two-stage stochastic model can be formulated in the following way.

$$
\begin{aligned}
& \min \left\{a^{T} x+\sum_{s \in S} p^{s} \times b^{s^{T}} \times y^{s}+\lambda \times \sigma\left(y^{1}, \ldots, y^{s}\right)+\omega \times \rho\left(\delta^{1}, \ldots, \delta^{s}\right)\right\} \\
& A x=c \\
& B^{s} x+C^{s} \times y^{s}+\delta^{s}=d^{s}, s \in S \\
& x, y^{s} \geq 0, s \in S
\end{aligned}
$$

Since with the shortage of any item in the worst-case scenarios, it is possible to supply these items from other suppliers at a higher unit price (however, one should not normally rely on them), the mentioned equation is changed into the following equation. The equation below shows that the required amount in the production or supply process must be supplied separately.

$$
\sum_{g, q, m} Z_{j g q m n t s}+\delta_{j n t s} \geq \text { demand }_{j n t s} \quad \forall j, n, t, s
$$

In addition, the term $\rho\left(\delta^{1}, \ldots, \delta^{S}\right)$ is changed into the following form:

$$
\rho\left(\delta^{1}, \ldots, \delta^{s}\right)=\sum_{j} \sum_{n} \sum_{t} \sum_{s} p^{s} \times E C_{j} \times \delta_{\text {jnts }}
$$

where $E C_{j}$ is the unit price of supplying goods from suppliers from whom purchase is not usually made. The presented models above consider variability symmetrically so that they take both negative and positive deviations into account in the calculations.

\section{RESULTS \\ 3.1 Data}

The data used for this model have been presented using the assessments of experts at Sabah Company. The used uncertain parameter is the demand parameter. The demand parameter depends on the number of retailers, the number of product variations, and time periods.

\subsection{The Results of the Sample Average Approximation Method}

The difference between these values has been practically considered as the value of the stochastic solution (VSS). VSS is calculated as follows: 
VSS $=\overline{\bar{f}}_{N}(\bar{X})-\overline{\bar{f}}_{N}\left(X^{\text {MeanValue }}\right)$

where, $\overline{\bar{f}}_{N}(\bar{X})$ is practically the middle value between the upper and lower bounds for the objective function obtained by the sample average approximation method for
$\bar{X}$ and $\overline{\bar{f}}_{N}\left(X^{\text {MeanValue }}\right)$ represents the value of the objective function for the problem with the average parameter. The comparative values for $\bar{X}^{1000}, \bar{X}^{2000}, \bar{X}^{3000}$, and $X^{\text {MeanValue }}$ can be observed in Tab. 1 .

Table 1 The values of the stochastic model solutions

\begin{tabular}{|c|c|c|c|c|}
\hline & $X^{\text {MeanValue }}$ & $\bar{X}\left(M=150, N=100, N^{\prime}=1000\right)$ & $\bar{X}\left(M=150, N=200, N^{\prime}=2000\right)$ & $\bar{X}\left(M=150, N=300, N^{\prime}=3000\right)$ \\
\hline OF & 1598974243 & 1495321887 & 1495403179 & 1495411159 \\
\hline VSS & - & 103652356 & 103571064 & 103563084 \\
\hline
\end{tabular}

As is shown in Tab. 1, using the stochastic model, the solutions improve by about $6.92 \%$ compared to the model with average parameters.

\subsection{Sensitivity Analysis of the Effect of Fuel Price on the Empty Capacity of the Vehicles}

Fig. 1 shows the model behaviour for different values of the coefficient of increase of fuel price (CIFP).

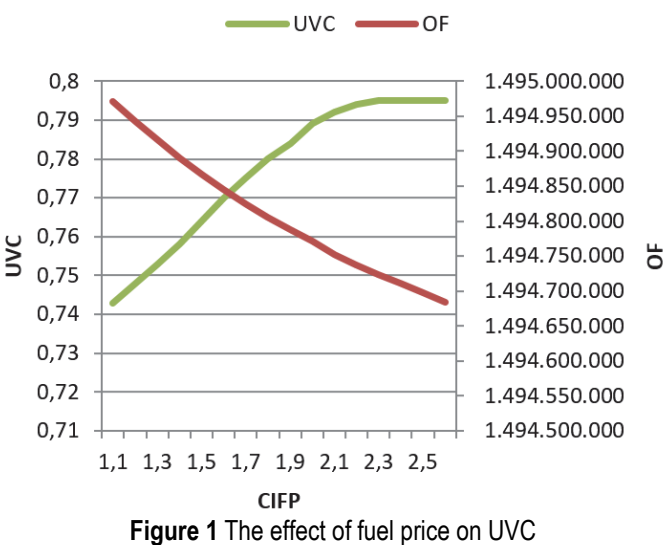

\subsection{Sensitivity Analysis of the Effect of Reduced Product Price with the Increase of Age}

Fig. 2 shows the behavior of the objective function of the problem and the percentage of the perished product with the decrease of selling price for different values of the coefficient of decrease of selling price (CDSP).

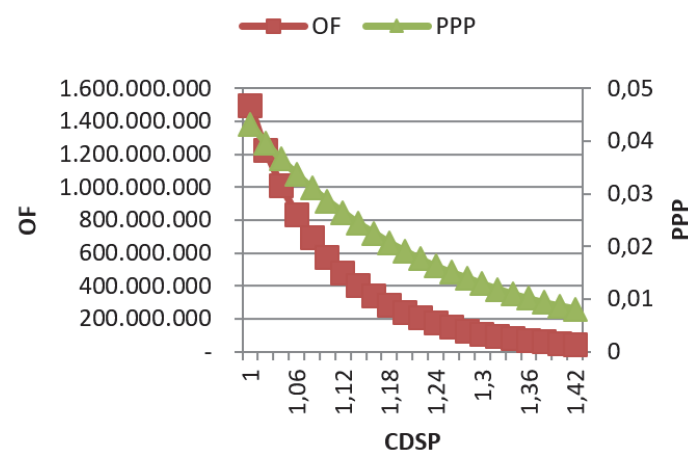

Figure 2 The effect of CDSP on PPP with the increase of age

It is clear that as CDSP increases, the value of the profit function decreases. On the other hand, the value of the perished product also decreases as CDSP increases. This behavior indicates that the percentage of perished product (PPP) will increase as profit margin increases in a system producing perishable items.

\subsection{Analysis of the Robust Two-Stage Stochastic Model}

In the following section, PLDM and PLDT criteria are investigated separately and a comparison of them is presented as well.

\subsubsection{The PLDM Criterion}

The different parts of the objective function are shown in this section. The objective function includes 7 main parts for the initial model, 1 part for the variability measure, and 1 part for the model robustness criterion. The main parts for the two-stage initial model include the sales revenues, supply cost, setup cost, vehicle supply cost, production cost, vehicle cost, and holding cost. It is clear that the value of the variability index PLDM practically decreases as the value of $\lambda$ which represents the importance of solution robustness increases. This can be observed in Fig. 3.

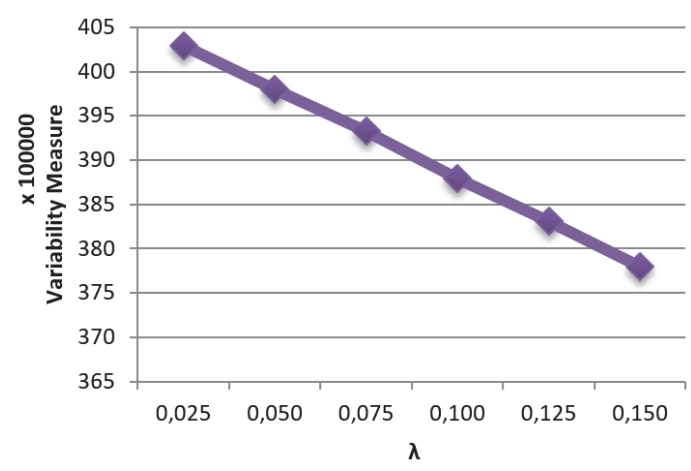

Figure 3 The relationship between $\lambda$ and the variability measure

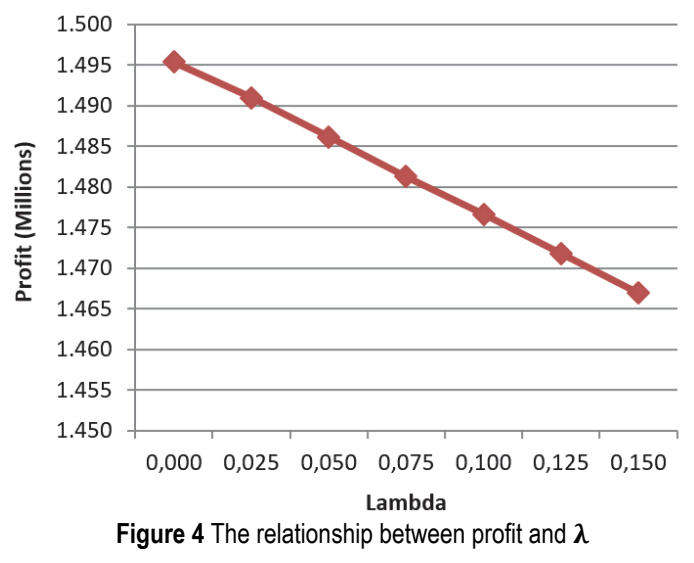


Fig. 4 shows the relationship between profit and $\lambda$. It is clear that as $\lambda$ increases, profit decreases. It is obvious that the amount of profit must practically decrease to reduce variability in the proposed model.

\subsubsection{The PLDT Criterion}

Fig. 5 shows the balance between the PLDT variability measure and the expected objective function for different values of $R^{*}$. It can be observed that a higher variability is obtained for the objective function as the value of $R^{*}$ increases. If the first priority of the decision maker is to increase profit, he/she must use a lower value for $R^{*}$. On the other hand, since the increase in the value of $R^{*}$ leads to the consideration of more scenarios in the variability calculations, the numerical value of the variability index increases, while the decrease of the $R^{*}$ value leads to the consideration of variability for a fewer number of scenarios.

\subsubsection{Comparison of the PLDM and PLDT Measures}

In this section, a comparison of the results obtained from the PLDM and PLDT models is presented so that the decision maker can practically choose one of the presented models. As was noted in previous sections, the PLDM model is only set by the parameter $\lambda$, while the PLDT model is set by parameters $\lambda$ and $R^{*}$.

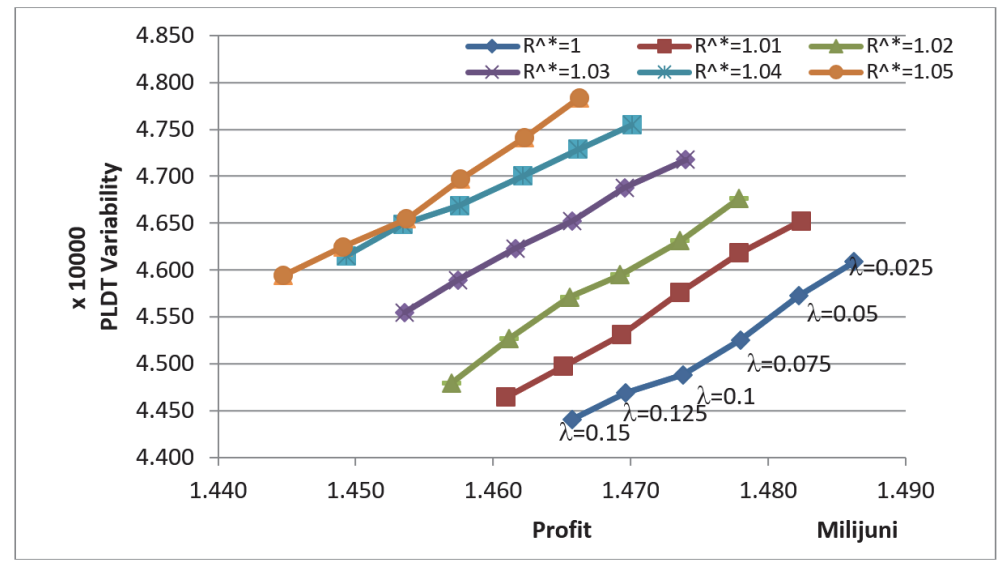

Figure 5 The mutual effect of the expected objective function and the PLDT variability measure

As is expected, since the PLDM model is practically dependent on mean, while the PLDT model is dependent on $R^{*}$, the freedom degree of the variability measure is higher for the PLDM model resulting in a higher reduction rate in the variability measure for a variation in a certain value of $\lambda$. Therefore, if the decision maker's main objective is to reduce variability, he/she must use the PLDM model with the maximum value of $\lambda$, as is shown in Fig. 6.

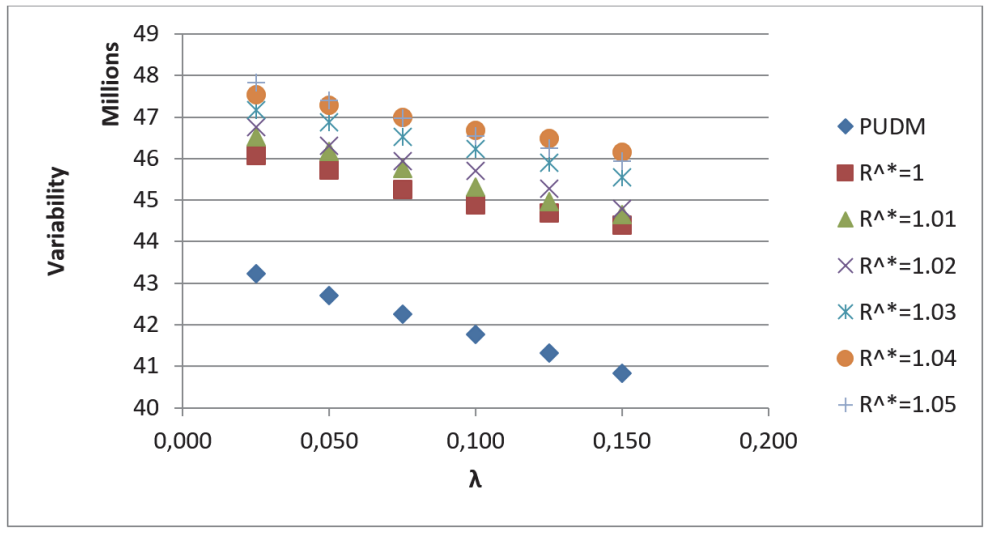

Figure 6 The comparison of the variability of the studied models versus the different values of $\lambda$

\section{CONCLUSION}

In this study, the three-level supply chain model for perishable items with different shelf lives was investigated and the sales revenue and holding cost could be determined according to the shelf lives of the products. The production and distribution centers could deliver fresh or old products to the customers according to the fresher first, older first, or free policies. However, the policy of production and distribution centers was free. The production rate was enough to meet customer demand. The proposed model was implemented in a two-stage structure. The decisions in the first stage determined the establishment of distribution centers and the other variables were determined in the second stage. To reduce the effect of different scenarios on the outputs of the problem, the two-stage model was developed to the robust model. Two variability criteria (PLDM and PLDT) were considered for the studied problem. This robust model can manage the variability of different scenarios based on the variability required in the 
problem. Since demand is not exactly clear even for different scenarios, besides the variability measures, different scenarios of the two-stage stochastic model were also examined in the fuzzy form and the corresponding fuzzy robust model was developed.

The results of these models showed that the supply cost, setup cost, vehicle supply cost, and production cost respectively accounted for $55 \%, 28 \%, 3 \%$, and $14 \%$ of the total costs of the chain. Moreover, the ratio of net profit margin to the total revenue of the supply chain derived from the division of the objective function by the revenue function was $15 \%$. Regarding free, fresher first, older first, and mixed policies, since the free policy imposes fewer constraints on the model, the decision maker gains more profit by the free policy than the other three policies. In the older first policy, given that the items with older shelf lives are delivered to the customers faster than the other items, the number of items which are expired is reduced, however, they give the decision maker the least revenue. In the fresher first policy, given that the fresh items are sold first and then older items are sold, the number of expired products is increased along the decision-making horizon. In return, the decision maker gains a higher revenue compared to the older first model.

\section{REFERENCES}

[1] Liu, G., Zhang, J., \& Tang, W. (2015). Joint dynamic pricing and investment strategy for perishable foods with pricequality dependent demand. Annals of Operations Research, 226(1), 397-416. https://doi.org/10.1007/s10479-014-1671-x

[2] Shah, N. H. (2017). Three-layered integrated inventory model for deteriorating items with quadratic demand and two-level trade credit financing. International Journal of Systems Science: Operations \& Logistics, 4(2), 85-91. https://doi.org/10.1080/23302674.2015.1075078

[3] Onggo, B. S., Panadero, J., Corlu, C. G., \& Juan, A. A. (2019). Agri-food supply chains with stochastic demands: A multi-period inventory routing problem with perishable products. Simulation Modelling Practice and Theory, 97, 101970. https://doi.org/10.1016/j.simpat.2019.101970

[4] Yang, P. C. \& Wee, H. M. (2002). A single-vendor and multiple-buyers production-inventory policy for a deteriorating item. European Journal of Operational Research, 143(3), 570-581. https://doi.org/10.1016/S0377-2217(01)00345-9

[5] Rau, H., Wu, M. Y., \& Wee, H. M. (2003). Integrated inventory model for deteriorating items under a multiechelon supply chain environment. International journal of production economics, 86(2), 155-168. https://doi.org/10.1016/S0925-5273(03)00048-3

[6] Yavari, M. \& Geraeli, M. (2019). Heuristic method for robust optimization model for green closed-loop supply chain network design of perishable goods. Journal of Cleaner Production, 226, 282-305. https://doi.org/10.1016/j.jclepro.2019.03.279

[7] Seyedhosseini, S. \& Ghoreyshi, S. (2014). An integrated model for production and distribution planning of perishable products with inventory and routing considerations. Mathematical Problems in Engineering, 2014(10), 1-10. https://doi.org/10.1155/2014/475606

[8] Yu, Y., Huang, G. Q., Hong, Z., \& Zhang, X. (2011). An integrated pricing and deteriorating model and a hybrid algorithm for a VMI (vendor-managed-inventory) supply chain. IEEE Transactions on Automation Science and Engineering, 8(4), 673-682.
https://doi.org/10.1109/TASE.2011.2140371

[9] Shaabani, H. \& Kamalabadi, I. N. (2016). An efficient population-based simulated annealing algorithm for the multi-product multi-retailer perishable inventory routing problem. Computers \& Industrial Engineering, 99, 189-201. https://doi.org/10.1016/j.cie.2016.07.022

[10] Hsu, C. I., Hung, S. F., \& Li, H. C. (2007). Vehicle routing problem with time-windows for perishable food delivery. Journal of food engineering, 80(2), 465-475. https://doi.org/10.1016/j.jfoodeng.2006.05.029

[11] Seyedhosseini, S. M., Mahdavi Mazdeh, M., Makui, A., \& Ghoreyshi, S. M. (2016). An inventory routing problem for perishable products with stochastic demands and direct deliveries. International Journal of Industrial Engineering \& Production Research, 27(1), 21-30.

[12] Chao, C., Zhihui, T., \& Baozhen, Y. (2019). Optimization of two-stage location-routing-inventory problem with timewindows in food distribution network. Annals of Operations Research, 273(1-2), 111-134. https://doi.org/10.1007/s10479-017-2514-3

[13] Khalili-Damghani, K., Abtahi, A. R., \& Ghasemi, A. (2015). A new bi-objective location-routing problem for distribution of perishable products: evolutionary computation approach. Journal of Mathematical Modelling and Algorithms in Operations Research, 14(3), 287-312. https://doi.org/10.1007/s10852-015-9274-3

\section{Contact information:}

Shima HARATI, PhD. student

(Corresponding author)

Department of Industrial Engineering,

South Tehran Branch, Islamic Azad University, Tehran, Iran

Emad ROGHANIAN, Associate Professor

Department of Industrial Engineering,

K. N. Toosi University of Technology, Tehran, Iran

Ashkan HAFEZALKOTOB, Assistant Professor

Department of Industrial Engineering,

South Tehran Branch, Islamic Azad University, Tehran, Iran

Amir Abbas SHOJAIE, Assistant Professor

Department of Industrial Engineering,

South Tehran Branch, Islamic Azad University, Tehran, Iran 